\title{
How to cultivate the intercultural communicative competence of college students in College English Teaching

\author{
Maohua SUN
}

Foreign Language Department, Dalian Jiao Tong University, Liaoning Province, China

I summer_817@163.com

Keywords: cross cultural communicative competence, College English teaching methods, College Students.

\begin{abstract}
. with the economic globalization and the frequent communication among people in different backgrounds, the ability of intercultural communication is becoming more and more important. This paper is mainly about the concept, the aspects and importance of intercultural communication. From the perspective of College English teaching, I will talk about how to cultivate college students' intercultural communicative competence.
\end{abstract}

\section{如何在大学英语教学中培养大学生的跨文化交际能力}

\author{
孙茂华 \\ 大连交通大学外国语学院, 大连, 辽宁省, 中国 \\ Isummer_817@163.com
}

关键词：跨文化交际能力, 大学英语教学方法, 大学生

中文摘要. 随着经济全球化, 不同背景的人们之间频繁交往, 跨文化交际能力越来越重要。 本文主要讲述跨文化交际的概念, 所包含的方面及其重要性, 从大学英语教学的角度出发讲 述如何培养大学生的跨文化交际能力。

\section{1. 引言}

随着我国对外开放和国际交流的发展, 我们清楚地认识到了跨文化交际的重要性。时代 的变化和要求使许多外语教育者对之表现出浓厚的兴趣, 越来越多的学者都在研究如何提高 跨文化交际能力。本文作者从其概念出发, 诠释了其组成部分以及大学生提高跨文化交际能 力的重要性, 最后作者提出了如何在大学英语教学中提高大学生的跨文化交际能力

\section{2. 跨文化交际的概念}

跨文化交际能力是跨文化交际学科的重要概念, 其内涵非常丰富, 学者们对这一概念做 了大量探讨, 并给出了不同的解释: (1) Spitzberg (2000:375) 将跨文化交际能力广义地视为 在某一特定语境中恰当和有效的行为; (2) Luatig和L（2007）认为跨文化能力由语境、得 体性与有效性以及知识、动机与行为组成; (3) Spencer-0atey和Frank1in (2009:51) 的定义 则是 “不同文化背景的交际者实施有效得体的言语或非言语交际行为及处理交际行为所产生 的心理问题和交际后果的能力; (4) Ting Toomey（2007:261) 认为跨文化交际能力是将理论和 实践相整合, 使交际者能够敏感而有意识地使用跨文化知识进行交流的方法。西方学者们的 界定多从社会学、心理学和交际学进行宏观表述。Wiseman (2004:192) 总结前人的研究成果, 
认为经过数十年研究, 学界逐渐达成共识, 跨文化交际能力特指能恰当而有效地与来自不同 文化背景的人们交流所需的知识、动机和技巧。

对于跨文化交际能力的具体内容，学者们提出了很多看法。有的学者认为跨文化交际能 力包括耐心、容忍、好奇、灵活性、诚实、尊敬对力、敏感、开放、不轻易下结论、自我控 制、倾听对力、适应性、移情、利-交能力、语言技能等在跨文化研究领域, 学者们对于跨文 化交际能力的主要组成部分 (components) 已达成一定共识LustiKocstcr ( 2007:69) 认为：“跨 文化能力需要足够的知识、合适的动机以及训练有素的行动单凭这些要素中的任何一个都小 足以获得跨文化能力” 也就是说, 知识、动机和行动三者结合才能构成跨文化能力Imxhori 和Lxnigxn（ 1989：277) 的观点是： “跨文化交际能力指的是外来者和本国人在他们的交际中 具各恰当的动机、知识和技能水平，这些要素引向有效的相互关系” 贾玉新 $1997: 480)$ 认为: “有效的跨文化交际能力至少由基本交际能力系统、情感和关系能力系统、情节能力系统和 交际力略能力系统组成” 杨盈和庄恩平 (2007：20) 认为： “跨文化交际能力由全球意识系统、 文化调适能力系统、知识能力系统和交际实践能力系统共同组成, 它们相互交织、密小可分, 共同构成跨文化交际能力的框架” 张红玲 (2007：70) 的看法是: “跨文化交际能力可以定义为: 掌握一定的文化和交际知识, 能将这些知识应用到实际跨文化环境中, 并且在心理上不惧怕, 主动、积极、愉快地接受挑战, 对不同文化表现出包容和欣赏的态度” 毕继万 (2005: 66) 认 为: “跨文化交际能力是在跨文化交际环境中由语言交际能力、非语言交际能力、语言规则和 交际规则转化能力以及文化适应能力组成的必各综合能力” 尽管不同学者对于跨文化交际能 力包含的要素观点不一, 但是从他们所列的要素中可以归纳出共同的部分, 即认知、感情 (态 度) 和行为这三个层而的能力在认知层面, 学者们指出要进行成功的跨文化交际, 交际者不仅 需要一般文化的知识, 还需要具各关于本国和其他国家的政治、经济、地理、历史、人文、 宗教、习俗等各力而的知识感情层而包括交际者对于文化差异的敏感、对于不同文化的包容、 对于自己文化的深刻理解以及对于其他文化的尊重等行为层而主要是指交际者的各种能力, 如语言能力, 这无疑是十分重要的、非语言能力、变通能力、处理人际关系的能力、心理调 适能力、适应环境的能力以及在异文化环境中做事的能力等。

\section{3. 培养大学生跨文化交际能力的重要性}

一种简便易行的排版方式为: 首先, 将论文内容粘贴至本模板; 其次, 格式刷所粘贴的 内容; 最后, 删除模板原有内容。如此, 可保持纸型、页面设置、字体、行间距等格式不便。 随着世界经济和教育全球化不断深入, 来自不同国家, 不同种族, 不同民族的人与人之间的 交流达到了前所未有的水平语言本身就是文化的载体。不同文化背景造成人们说话方式或行 为习惯不尽相同，交际中不可避免地出现大量的矛后或冲突 。

当今随着英语的重要性得到普遍认可, 大学英语教学就有了更高的日标和更高的要求, 不仅要学好英语这门语言本身, 还需要我们了解与语言相联系的语境, 语境通常包括文化的 内容这就要求教师在语言教学的同时应尽可能加强文化意识的渗透, 在教授语言的同时, 教 授文化知识提高学生对英美文化的敏感性与洞察力, 培养学生的跨文化意识和跨文化能力, 使学生能够真正做到学以致用, 进一步推动我国英语教育事业向纵深发展, 以便他们更能正 确地使用语言, 选择恰当的交流方式减少跨文化交流中的障碍。 


\section{4. 在大学英语教学中培养学生的跨文化交际能力的方法及措施}

\section{1 提高大学英语教师的整体素养, 培养大学生的跨文化交际能力对教师和学生都提出了新 的要求}

首先，大学英语教师要不断地提高自身的文化素养通过了解英语国家的政治，经济，历 史, 地理, 文艺, 宗教和利一会习俗等积累英美国家的文化背景常识另外英语教师要不断地 阅读和英语教学有关的跨文化交际力而的专著汲取新的知识和新的教学力法, 结合自身和所 教学生的实际情况把自己所学的应用到课堂教学中去教师还可以通过参加相关专业的学术会 议, 做访问学者, 短期进修来提高自己, 从而调整教学, 改善教学效果教师要在文化输入力 而不断地加强深度和力度, 使学生充分意识到本族语与英语及其所属文化的差异, 从而在交 流中避免误解。

\section{2 在大学英语教学活动中学生是主体}

我国大学英语教学长期以来局限于传统的教学模式, 过分强调教师在教学活动中的主导 地位, 学生的情感和个性受到压抑, 在语言实践中缺乏自信, 英语应用能力差。所以大学英 语教师在大学英语课堂培养学生的跨文化交际能力就应该重视学生在教学活动中的主体地位 和主动精神, 注重指导学生的课堂活动, 启发学生的思维, 使学生能够灵活运用自己所学的 语言文化进行跨文化交际活动, 过后让学生深刻总结自己的经验以及小足之处, 为以后成功 的进行跨文化交际打下基础。

\section{3 增加大学生跨文化交际活动的机会}

实际上, 跨文化交际能力培养小仅需要教学环节的精心设计, 还需要增加学生的课外实 践机会比如:在认知层而, 一般采取讲课、阅读有关材料、使用音像制品、网络教育等力式进 行在感情层而, 力式多种多样, 包括典型案例分析、与小同文化背景的人互动、短期的国外 学习或上作日前, 有些外语院校己经有国内学习和国外学习相结合的安排, 这种做法除了有 利于培养语言能力, 还对跨文化交际能力培养起着重要作用。

\section{5. 大学英语跨文化交际能力培养途径}

如果将跨文化教学等同于大学英语跨文化交际能力培养的全部内容, 无疑过于简单化。 跨文化交际能力由知识、动机和技能三部分组成, 要求交际者同时具备认知、情感和行为层 面的能力, 是一个很高的目标 (胡文仲, 2013), 目前大学英语教学最主要是课堂教学和课外 主题活动两种途径。

通过课堂授课实现跨文化交际能力培养涉及培养目标确定、教学内容和材料选择、教学 方法和教学活动设计、评估测试方式更新。把跨文化教学融入语言教学框架中, 既要传授语 言知识, 还要传播语言背后文化规约、交际规范的隐性知识。教师要分析确定学生的起点水 平, 层级考虑学习内容的难易程度和深浅, 界定学生知识掌握的程度并作出具体要求。社会 和学校大环境的配合与支持是大学英语跨文化交际能力培养必不可少的因素。培养跨文化交 际能力，可从以下具体途径来进行：

\section{1 加强师资培训}

外语教师大部分都是文学和语言学背景, 没有系统学习过跨文化教育理论, 为实现外语 语言教学向跨文化教学转变, 大学英语教师有必要研读学习跨文化交际理论著作。

\section{2 开发优质教材}

教材是教学的核心, 国内各大出版社出版的大学英语教材得到广大师生的认可, 呈现多 本化、“百花齐放” 的样态, 教材从一本书向系列化发展, 形式从书本向多媒体课件延伸。 


\section{3 培养双向跨文化意识}

大学英语教学长期以来习惯于学习英美文化, 对学生的跨文化意识培养也集中于如何使 学生了解英美文化。随着我们国家世界影响力的不断扩大, 同时出于对外传播中国文化的需 要, 培养学生的双向跨文化意识成为必然。跨文化意识培养中应该增加中国文化内容, 增强 我国文化的对外表述能力, 弘扬民族文化, 增强文化平等意识, 我国英语教育跨文化意识双 向成长是由国家发展战略态势所决定的 (赵海燕, 2013)。

\section{4 重视实践培训项目}

从本质上讲, 跨文化交际能力是一种行为层面上的能力, 缺少实践的支撑, 也就违背了 它的基本属性, 没有生命力和应用价值 (张卫东杨莉, 2012:8)。利用第二课堂, 丰富课外实 践活动, 加强国际交流, 把学生置身于真实的跨文化交流互动中去,

或者应用现代网络、多媒体技术, 带领学生进入虚拟的跨文化交流平台, 如通过emai1结交异 国搭档, 使学生直接参与跨文化实时互动, 这些做法都对大学英语的跨文化交际能力培养起 着重要作用。将跨文化交际能力培养作为大学英语教学的重要维度深化了大学英语课程 的内涵, 使英语教学本身更有意义, 跨文化教学融入大学英语教学能够促进大学英语课程工 具性与人文性的有机融合, 将社会主义核心价值观有机融入大学英语课程, 使大学英语符合 国家、社会发展的时代需要, 并更好地服务于学生个人发展需求。

\section{6. 结论}

总之, 随着世界经济全球化, 外语教学改革也在不断地深入, 作为大学英语教师不仅要 使学生掌握一门外语, 而且还应该不断地培养与提高大学生的跨文化交际能力, 使他们在跨 文化交际中能够进行有效的跨文化交流。

\section{References}

[1] Chen Yu Lu's 2012 cross cultural communication ability: the new demand for cultivating the top innovative talents. China's higher education.

[2] Jia Yuxin 1997 cross cultural communication [M] "Shanghai: Shanghai Foreign Language Education Press.

[3] $\mathrm{Hu}$ Wenzhong Gao Yi Hong 1997 foreign language teaching and culture [M] "Changsha: Hunan Education Press.

[4] Liu Yandong 2014, to promote the improvement of language skills and promote the development of human civilization and social progress. The speech at the opening ceremony of the world language conference [R] Suzhou: world language conference.

[5] Research on the training system of English intercultural communicative competence of the 2012 University of Wei Ling, and the study of open education in the field of practice (1): 118. 123.

[6] Wen Qiufang 1999 spoken English testing and teaching areas: Shanghai Foreign Language Education Press.

[7] Wang flame 2012 research field of College English teaching based on cross-cultural communication: Wuhan: Huazhong University of Science and Technology.

[8] Yang Ying, Zhuang Siping 2007 to build a foreign language field of intercultural communicative competence in foreign language teaching, (4) 13-21. 
[9] Zhang Weidong Yang Li 2012 cross cultural communication ability system construction based on foreign language education perspective and empirical research method field foreign language field, (2): 8 to 16 .

[10]Zhao Haiyan 2013 on intercultural awareness in English education in China two-way growth Tian Zhongguo Journal of education, (11): 67-69. 